\title{
Joint Rate and Power Control Using Game Theory
}

\author{
Madhusudhan R. Musku, Anthony T. Chronopoulos, and Dimitrie C. Popescu \\ Student Member, IEEE Senior Member, IEEE Senior Member, IEEE \\ Electrical Engineering and Computer Science Departments \\ The University of Texas at San Antonio \\ 6900 N Loop 1604 W, San Antonio, TX 78249 \\ Email:mmusku@lonestar.utsa.edu, atc@cs.utsa.edu, and dimitrie.popescu@utsa.edu
}

\begin{abstract}
Efficient use of available resources in next generation wireless systems require control of both data rate and transmitted power for mobile terminals. In this paper the problem of joint transmission rate and power control is approached from the perspective of non-cooperative game theory, and an algorithm for joint rate and power control is presented. A new utility function for mobile terminals is defined, and a detailed analysis of the existence and uniqueness of Nash equilibrium for the non-cooperative joint transmission rate and power control game is presented. The utility function depends on the signal to interference ratio (SIR), and can be adjusted to provide the desired Quality of Service $(Q 0 S)$ requirement. Numerical simulations that compare the proposed algorithm with alternative algorithms developed using game theory are also presented in the paper.
\end{abstract}

\section{INTRODUCTION}

Next generation wireless systems will provide a wide range of services for mobile users, from multimedia transmissions performed in real time to transmission of data that can tolerate delay and which is not performed in real time. In order to provide these heterogeneous services, efficient use of system resources in this case requires control of both data rate and transmitted power control. This requirement implies the need for joint rate and power control for mobile terminals, as opposed to only power control on which [1], [2], [4], [7], [8], [10] mainly focus on.

The main goal of power control is to provide adequate quality for the signal of each mobile terminal at the receiver without causing unnecessary interference to signals transmitted by other mobile terminals. Power control helps also to extend the battery life of mobile terminals by ensuring that these transmit at the minimum power level necessary to achieve the required QoS. Game-theoretic approaches to power control [1], [2], [4], [7] describe QoS for mobile terminals using utility functions. The concept of utility is commonly used in microeconomics and game theory to denote the level of satisfaction of a decision-maker with specific products or services as a result of its actions. Game theory is used to model interactions among self-interested users and predict their choice of strategies to optimize utility functions until a Nash equilibrium is reached, where no user can further increase its corresponding utility through individual action. Utility functions used for wireless systems depend usually on both the SIR and the transmitted power of a given terminal. In [2], [7] the utility function is dependent on low-level system parameters like modulation, coding and packet size. In this case the SIR at equilibrium depends on the length of the packet, and implies that users should increase the packet size to achieve higher equilibrium SIRs. In [4] a different utility function which depends on the channel capacity and decouples lower layer decisions like modulation and coding is proposed.

Research in the area of joint rate and power control is emerging and related work can be found in [5], [9]. In [5], joint rate and power control is approached from a gametheoretic perspective, and modeled as two distinct games. The algorithm in [5] requires that all terminals find first the rate of transmission, and then apply power control to allocate the powers. A more comprehensive algorithm for joint rate and power control is the one proposed in [9]. In this case, terminals closer to the base station achieve higher rates at lower powers, while terminals farther away from the base station transmit at full power with very low rates.

In our paper we propose a joint rate and power control algorithm which finds the optimal rate of transmission and allocates the power required to transmit in the same step. This is different from previous approaches, and implies a simpler implementation with lower computational complexity. The paper is organized as follows: in Section II we describe the system model and introduce a new utility function to be used for joint rate and power control. In Section III we formulate joint rate and power control as a non-cooperative game, and investigate existence and uniqueness of a Nash equilibrium solution for this game. In Section IV we present numerical results obtained from simulations that compare the proposed algorithm with the algorithm in [9]. Conclusions and directions for future work are presented in Section V.

\section{System Model and Utility Function}

We consider a single-cell of a CDMA wireless communication system with $N$ mobile terminals (users) transmitting data to the same base station, in which the SIR corresponding to a given user $j$ is expressed as [7]

$$
\gamma_{j}=\frac{W}{r_{j}} \frac{h_{j} p_{j}}{\sum_{k=1, j \neq k}^{N} h_{k} p_{k}+\sigma^{2}} \quad j=1,2, \ldots, N
$$

where $W$ is the available (spread-spectrum) bandwidth, $\sigma^{2}$ is the Additive White Gaussian Noise (AWGN) power spectral 
density at the receiver, $h_{j}$ is the path gain of user $j$ to the base station, and $r_{j}$, respectively $p_{j}$, are the transmission rate, respectively power, of user $j$. This expression assumes that users in the CDMA system are assigned pseudorandom noise (PN) sequences, and that conventional matched filter detectors are used at the receiver [7].

The objective of each user is to optimize its transmission rate and power in a distributed manner, such that its corresponding utility is maximized. In general, utility functions used for wireless systems depend on power and SIR, and must satisfy two main properties [7]:

1) For fixed transmit powers, the utility increases with the increase in the SIR of the terminal

2) For fixed SIR, the utility decreases as the transmitted power increases.

For rate control the utility function must also satisfy:

3) For fixed transmission rates, the utility increases with the increase in the SIR of the terminal.

4) For fixed SIR, the utility increases as the rate increases. These requirements prompted us to consider the following utility function for given user $j$ in our game theoretic approach to joint rate and power control

$$
u_{j}=\frac{r_{j} \ln \left(K \gamma_{j}\right)}{p_{j}}[\mathrm{bits} / \mathrm{J}]
$$

where $K$ is a parameter that depends on the QoS requirements. The utility expression in equation (2) assumes that users transmit data in frames (or packets) of length $M$, and takes $K$ to be a function of the SIR

$$
K=e^{g\left(\gamma_{j}\right)} / \gamma_{j}
$$

where $g\left(\gamma_{j}\right)=\left[1-P_{e}\left(\gamma_{j}\right)\right]^{M}$ is the Frame Success Rate (FSR) with $P_{e}\left(\gamma_{j}\right)$ being the bit-error rate and $M$ the length of the packet. This implies that $\ln \left(K \gamma_{j}\right)=g\left(\gamma_{j}\right)$ and the term in the numerator of equation (2) is the product between user $j$ 's transmission rate $r_{j}$ and its corresponding FSR $g\left(\gamma_{j}\right)$, and defines user $j$ 's throughput $T_{j}=r_{j} g\left(\gamma_{j}\right)$. Thus, the utility function in equation (2) can be regarded as the ratio of user $j$ 's throughput $T_{j}$ to the transmitted power $p_{j}$. We note that the utility function is not defined when transmit power $p_{j}=0$, and in order to avoid this situation we impose a lower bound $\hat{p}$ on all user transmit powers, that is $p_{j} \geq \hat{p}$, which is the minimum power level for transmission for all users in the system.

In this context, the objective of each user in the system is to adapt its transmitted power and rate in a distributed manner, such that its corresponding utility is maximized.

\section{Formulation AS A NON COOPERATIVE GAME}

Let $G=\left[\mathcal{N},\left\{P_{j}, R_{j}\right\},\left\{u_{j}(\cdot)\right\}\right]$ denote the non-cooperative rate and power control game (NRPG), where $\mathcal{N}=$ $\{1,2, \ldots, N\}$ is the index set for the active mobile users in the cell, $P_{j}$ is the strategy set of user powers, $R_{j}$ is the strategy set of user rates, and $u_{j}(\cdot)$ is the utility function. Each user selects a rate $r_{j} \in R_{j}$ and a power $p_{j} \in P_{j}$. Let the rate vector $\mathbf{r}=$ $\left(r_{1}, r_{2}, \ldots, r_{N}\right)^{T} \in R^{N}=R_{1} \times R_{2} \times \ldots \times R_{N}$, power vector $\left.\mathbf{p}=\left(p_{1}, p_{2}, \ldots, p_{N}\right)^{T} \in P^{N}=P_{1} \times P_{2} \times \ldots \times P_{N}\right)$ (where $T$ represents the transpose operator) denote the outcome of the game in terms of selected rate and power levels of all the users. The utility of user $j$ when it transmits at rate $r_{j}$ by expending power $p_{j}$ is given in equation (2). We assume that the strategy spaces $R_{j}$ and $P_{j}$ of each user are compact and convex sets with maximum and minimum constraints. For any given user $j$ we consider strategy spaces the closed intervals $R_{j}=\left[r_{j}, \overline{r_{j}}\right]$ and $P_{j}=\left[\underline{p_{j}}, \overline{p_{j}}\right]$, where the minimum power $p_{j} \geq \hat{p}$.

In a distributed rate and power control game, each user adjusts rate $r_{j}$ and power $p_{j}$ in order to maximize the utility $u_{j}$. Formally, the NRPG is expressed as

$$
\max _{r_{j} \in R_{j}, p_{j} \in P_{j}} u_{j}(\mathbf{r}, \mathbf{p}) \quad \text { for all } j \in \mathcal{N}
$$

where $u_{j}$ is given in (2) and $R_{j}=\left[r_{j}, \overline{r_{j}}\right]$ and $P_{j}=\left[p_{j}, \overline{p_{j}}\right]$ are the strategy spaces of user $j$. From the perspective of rate optimization, user $j$ 's strategy is to find a transmission rate $\tilde{r}_{j}$ in the rate strategy space $R_{j}$ which maximizes its utility $u_{j}$. The necessary condition for maximizing utility with respect to transmission rate $\left(\partial u_{j} / \partial r_{j}=0\right)$ implies

$$
\ln \left(K \gamma_{j}\right)-1=0
$$

From the perspective of power optimization, user $j$ 's strategy is to find a power level $\tilde{p}_{j}$ in the strategy space $P_{j}$ which maximizes its utility $u_{j}$. The necessary condition for maximizing utility with respect to power $\left(\partial u_{j} / \partial p_{j}=0\right)$ leads to the same condition for maximizing utility as in equation (5). Thus, maximum utility occurs under the same condition for both rate and power control.

The necessary condition for optimizing utility with respect to rate and power can also be used to obtain the value of the parameter $K$. By rearranging equation (5) we get

$$
K=\frac{e}{\tilde{\gamma}}
$$

where $\tilde{\gamma}$ is the target SIR, which can be assumed to be transmitted to each user by the base station. We note that the value of $K$ in (6) corresponds to FSR equal to 1, which is obtained when there are no errors in the transmission. However, this is not guaranteed in real systems, and the practical way of finding the value of $K$ is to equate $\ln (K \gamma)$ to a desired FSR $P_{c}$

$$
\ln (K \tilde{\gamma})=P_{c} \quad \Longrightarrow \quad K=\frac{e^{P_{c}}}{\tilde{\gamma}}
$$

and obtain the value of the target SIR $\tilde{\gamma}$ from equation (7) from the value of $K$ as

$$
\tilde{\gamma}=\frac{e^{P_{c}}}{K}
$$

The advantage of this procedure is that the target SIR can be determined by adjusting the value of $K$, a feature which was not available in previous work [4], [7], [9]. 


\section{A. The Nash Equilibrium}

A Nash equilibrium is a fixed point of a non-cooperative game where no user can increase the value of its utility function through individual action. For joint rate and power control we investigate Nash equilibrium solutions for the rate and power games defined in the previous section. We will show that a Nash equilibrium solution exists and is unique by using the theory of concave games [6]. We note that a non-cooperative game $G$ is called concave if all user utility functions are strictly concave with respect to their corresponding strategies [6].

According to [6] a Nash equilibrium exists in a concave game if the joint strategy space is compact and convex, and the utility function that any given player seeks to maximize is concave in its own strategy and continuous at every point in the product strategy space. In addition, if the weighted sum of the utility functions with nonnegative weights

$$
\sigma=\sum_{j=1}^{N} x_{j} u_{j}, \quad x_{j} \geq 0 \quad \forall j
$$

is diagonally strictly concave, then the Nash equilibrium point is unique. We note that the notion of diagonal strict concavity signifies in loose terms that an individual user has more control over its utility function than the other users have on it, and is checked using the pseudo-gradient of the weighted sum of utility functions [6].

A Nash equilibrium in rates is formally defined as

Definition 1: A rate vector $\mathbf{r}=\left(r_{1}, \ldots, r_{N}\right)$ is a Nash equilibrium of the NRPG $G=\left[\mathcal{N},\left\{P_{j}, R_{j}\right\},\left\{u_{j}\right\}\right]$ if, for every $j \in \mathcal{N}, u_{j}\left(r_{j}, \mathbf{r}_{-j}\right) \geq u_{j}\left(r_{j}^{\prime}, \mathbf{r}_{-j}\right)$ for all $r_{j}^{\prime} \in R_{j}$, where $\mathbf{r}_{-j}=\left(r_{1}, \ldots, r_{j-1}, r_{j+1}, \ldots, r_{N}\right)^{T}$ is the $N-1$ dimensional vector of user rates that does not contain user $j$ 's rate.

Theorem 1: A Nash equilibrium in transmission rates for game $G=\left[\mathcal{N},\left\{P_{j}, R_{j}\right\},\left\{u_{j}(\cdot)\right\}\right]$ exists and is unique.

Proof: In order to prove existence, we note that each user's strategy space $R_{j}$ is defined by all rates in the closed interval bounded by the minimum and maximum rates. Thus, the joint strategy space $R$ is a nonempty, convex, and compact subset of the Euclidean space $\Re^{N}$. In addition, the utility functions are concave with respect to user rates as can be seen from the second derivative test:

$$
\frac{\partial^{2} u_{j}}{\partial r_{j}^{2}}=\frac{1}{p_{j}} \frac{\partial \gamma_{j} / \partial r_{j}}{\gamma_{j}}=-\frac{1}{p_{j} r_{j}}<0 \quad \forall j \in \mathcal{N}
$$

which ensures existence of a Nash equilibrium.

In order to prove uniqueness, we follow [6] and define the weighted sum of user utility functions

$$
\mu(\mathbf{r}, \mathbf{x})=\sum_{j=1}^{N} x_{j} u_{j}\left(r_{j}, \mathbf{r}_{-j}\right)
$$

for which the pseudo-gradient is given by

$$
f(\mathbf{r}, \mathbf{x})=\left[x_{1} \nabla u_{1}\left(r_{1}, \mathbf{r}_{-1}\right), \ldots, x_{N} \nabla u_{N}\left(r_{N}, \mathbf{r}_{-N}\right)\right]^{T}
$$

The Jacobian of the pseudo-gradient with respect to $\mathbf{r}$ is the matrix $\mathbf{F}=\left(B_{j i}\right)_{1 \leq j, i \leq N}$ with elements

$$
B_{j i}= \begin{cases}x_{j} \frac{\partial^{2} u\left(r_{j}, \mathbf{r}_{-j}\right)}{\partial r_{j} \partial r_{i}}=-\frac{x_{j}}{p_{j} r_{j}}<0 & \text { for } j=i \\ x_{j} \frac{\partial^{2} u\left(r_{j}, \mathbf{r}_{-j}\right)}{\partial r_{j} \partial r_{i}}=0 & \text { for } j \neq i\end{cases}
$$

Thus, $\mathbf{F}$ is a diagonal matrix with all diagonal elements negative which implies that it is negative definite. This implies that $\left[\mathbf{F}+\mathbf{F}^{T}\right]$ is also negative definite, and according to Theorem 6 in [6] the weighted sum of the utility functions $\mu(\mathbf{r}, \mathbf{x})$ is diagonally strictly concave and the Nash equilibrium point with rates

$$
\tilde{r}_{j}=\arg \max _{r_{j} \in R_{j}} u_{j}\left(r_{j}, \mathbf{r}_{-j}\right) \quad \forall j \in \mathcal{N}
$$

is unique.

A Nash equilibrium in transmitted powers is defined formally as

Definition 2: A power vector $\mathbf{p}=\left(p_{1}, \ldots, p_{N}\right)$ is a Nash equilibrium of the NRPG $G=\left[\mathcal{N},\left\{P_{j}, R_{j}\right\},\left\{u_{j}\right\}\right]$ if for every $j \in \mathcal{N}, u_{j}\left(p_{j}, \mathbf{p}_{-j}\right) \geq u_{j}\left(p_{j}^{\prime}, \mathbf{p}_{-j}\right)$ for all $p_{j}^{\prime} \in P_{j}$, where $\mathbf{p}_{-j}=\left(p_{1}, \ldots, p_{j-1}, p_{j+1}, \ldots, p_{N}\right)^{T}$ is the $N-1$ dimensional vector of user power that does not contain user j's power.

Theorem 2: A Nash equilibrium in transmission powers for game $G=\left[\mathcal{N},\left\{P_{j}, R_{j}\right\},\left\{u_{j}(\cdot)\right\}\right]$ exists and is unique.

Proof: To prove existence we use a similar line of reasoning as before and note that the joint strategy space $P$ is a nonempty, convex, and compact subset of the Euclidean space $\Re^{N}$ since it is implied by closed intervals for user powers defined by minimum and maximum powers. The second derivative test in this case implies that the utility functions are concave with respect to user powers as well since

$$
\frac{\partial^{2} u_{j}}{\partial p_{j}^{2}}=r_{j}\left[\frac{2 \ln \left(K \gamma_{j}\right)-3}{p_{j}^{3}}\right]<0 \quad \forall j \in \mathcal{N}
$$

because the FSR is always less than 1, which implies that $\ln \left(K \gamma_{j}\right) \leq 1$. This ensures existence of a Nash equilibrium in powers for the NRPG.

In order to prove uniqueness we define now the weighted sum of user utility functions

$$
\sigma(\mathbf{p}, \mathbf{x})=\sum_{j=1}^{N} x_{j} u_{j}\left(p_{j}, \mathbf{p}_{-j}\right)
$$

for which the pseudo-gradient is given by

$$
f(\mathbf{p}, \mathbf{x})=\left[x_{1} \nabla u_{1}\left(p_{1}, \mathbf{p}_{-1}\right), \ldots, x_{N} \nabla u_{N}\left(p_{N}, \mathbf{p}_{-N}\right)\right]^{T}
$$

To show that $\sigma(\mathbf{p}, \mathbf{x})$ is diagonally strictly concave in this case we use the following lemma proved in [3].

Lemma 1: If each $u_{j}(\mathbf{p})$ is a strictly concave function in $p_{j}$, each $u_{j}(\mathbf{p})$ is convex in $\mathbf{p}_{-j}$ and there is some $\mathbf{x}>0$ such that $\sigma(\mathbf{p}, \mathbf{x})$ is concave in $\mathbf{p}$, then $\left[\mathbf{F}(\mathbf{p}, \mathbf{x})+\mathbf{F}^{T}(\mathbf{p}, \mathbf{x})\right]$ is negative definite, where $\mathbf{F}$ is the Jacobian of $f(\mathbf{p}, \mathbf{x})$. 
From (15), we know that $u_{j}(\mathbf{p})$ is strictly concave in $p_{j}$. In addition

$$
\left.\frac{\partial^{2} u_{j}}{\partial p_{i}^{2}}\right|_{i \neq j}=\frac{r_{j}}{p_{j}} \frac{h_{i}^{2}}{\sum_{k \neq j, i}^{N} h_{k} p_{k}+h_{i} p_{i}+\sigma^{2}}>0
$$

which implies that $u_{j}(\mathbf{p})$ is convex in $\mathbf{p}_{-j}$ as well. Since the second derivative is also negative

$$
\frac{\partial^{2} \sigma(\mathbf{r}, \mathbf{x})}{\partial p_{j}^{2}}=\sum_{j=1}^{N} x_{j} r_{j}\left[\frac{2 \ln (K \gamma)-3}{p_{j}^{3}}\right]<0
$$

then $\sigma(\mathbf{r}, \mathbf{x})$ is concave in $p_{j}$ and from Lemma 1 we have that $\left[\mathbf{F}+\mathbf{F}^{T}\right]$ is negative definite. Thus the weighted sum of utility functions $\sigma(\mathbf{r}, \mathbf{x})$ is diagonally strictly concave, and the Nash equilibrium point with powers

$$
\tilde{\mathbf{p}}=\arg \max _{p_{j} \in P_{j}} u_{j}\left(p_{j}, \mathbf{p}_{-j}\right) \quad \forall j \in \mathcal{N}
$$

is unique.

\section{B. The Joint Rate and Power Control Algorithm}

The condition which maximizes the utility given in equation (5) can be rearranged as

$$
\tilde{\gamma}=\frac{e}{K}
$$

The SIR at maximum utility can be written as

$$
\tilde{\gamma}_{j}=\frac{W}{\tilde{r}_{j}} \frac{h_{j} \tilde{p}_{j}}{\sum_{k=1, j \neq k}^{N} h_{k} p_{k}+\sigma^{2}}
$$

By substituting (22) in (21) and rearranging the terms, the equilibrium rate of user $j$ is written as

$$
\tilde{r_{j}}=\frac{W K}{e} \frac{h_{j} p_{j}}{\sum_{k=1, j \neq k}^{N} h_{k} p_{k}+\sigma^{2}}
$$

Similarly, the power of terminal $j$ at the equilibrium can be shown to be

$$
\tilde{p_{j}}=\frac{r_{j} e}{W K h_{j}}\left(\sum_{k=1, j \neq k}^{N} h_{k} p_{k}+\sigma^{2}\right)
$$

The joint rate and power control algorithm based on the NRPG is formally stated below:

\section{NRPG Algorithm}

1) Initialize power vector $p$ randomly at time $t_{0}$.

2) For all $j \in \mathcal{N}$ at time instant $t_{k}$

a) Update user $j$ rate using equation (23)

b) Update user $j$ power using equation (24)

3) If $\left\|\mathbf{p}\left(t_{k}\right), \mathbf{p}\left(t_{k-1}\right)\right\| \leq \epsilon$, then STOP and declare the Nash equilibrium as $p\left(t_{k}\right)$. Else, make $k=k+1$ and go to step 2 .
We note that even though equations (23) and (24) seem to imply that every terminal needs to have knowledge of all the other terminals in the system, in reality the term

$$
\left(\sum_{k=1, j \neq k}^{N} h_{k} p_{k}+\sigma^{2}\right)
$$

represents the interference plus noise experienced by user $j$ 's signal at the base station. This can be assumed to be transmitted to users by the base station through a feedback channel, so that it is available to users when they update transmission rates and transmit powers.

\section{Simulations}

We compare the Non-cooperative Rate and Power control (NRPG) algorithm with the non cooperative power control game (NPG) described in [7] as well as with the rate and power control algorithm described in [9]. In doing so, we take the same system parameters as given in [7] and [9]. In both setups all users are assumed to be stationary, and the propagation model employed has gains inversely proportional to the $4^{\text {th }}$ power of the distance $d_{j}$ (in meters) from the base station, that is $h_{j}=c / d_{j}^{4}$, with $c=0.097$.

In the first simulation we consider the setup in [7] with $N=9$ users located at distances $d=[310,460,570,660$, $740,810,880,940,1000]$ meters from the base station, and the following system parameters are considered:

- Spread spectrum bandwidth $W=10^{6} \mathrm{~Hz}$.

- AWGN power at the receiver $\sigma^{2}=5 \cdot 10^{-15}$ Watts.

- The maximum power of each user $\bar{p}=2$ Watts.

- The minimum power of each user $p=\hat{p}=10^{-4}$ Watts.

- The maximum and minimum rate constraints $\bar{r}=\underline{r}=$ $10^{4}$ are identical because in this case the algorithm is used only for power control.

We consider the target SIR equal to 12.42 (the equilibrium SIR obtained by the algorithm in [7]) and use equation (6) to calculate the value of the parameter $K=0.21886$.

In Figure 1, the equilibrium powers and utilities obtained by NRPG are compared with those obtained by the NPG in [7]. We note that the equilibrium powers are the same in both the algorithms for the same equilibrium SIRs.

In the second simulation we consider the setup in [9], with $N=11$ users at distances $d=[50,100,150,200,250,300$, $350,400,450,450,500]$ meters from the base station, and the following system parameters:

- Spread spectrum bandwidth $W=3.84 \times 10^{6} \mathrm{~Hz}$.

- AWGN power at the receiver $\sigma^{2}=10^{-15}$ Watts.

- The maximum power of each user $\bar{p}=0.2$ Watts.

- The maximum rate for all users $\bar{r}=96 \mathrm{kbps}$.

- The minimum rate for all users $\underline{r}=0 \mathrm{kbps}$.

In Figure 2, the equilibrium powers, rates and SIRs obtained by NRPG are compared with those obtained by the rate and power control algorithm described in [9]. We note that the equilibrium transmission rates and powers are identical for the proposed NRPG and for the algorithm in [9], with users 


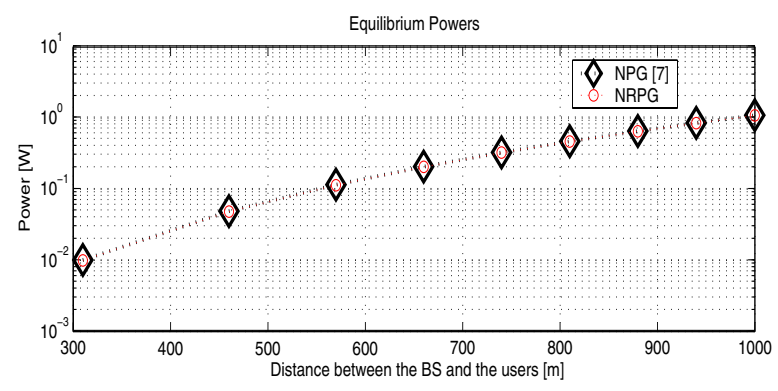

Equilibrium SIRs

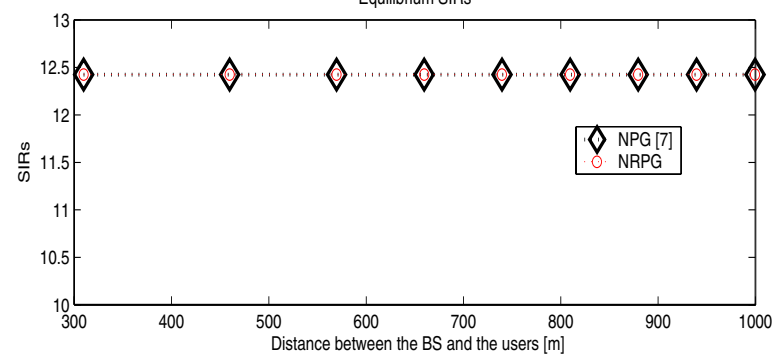

Fig. 1. Equilibrium Powers and SIRs of NPG ([7]) and NRPG (with constant transmission rates)
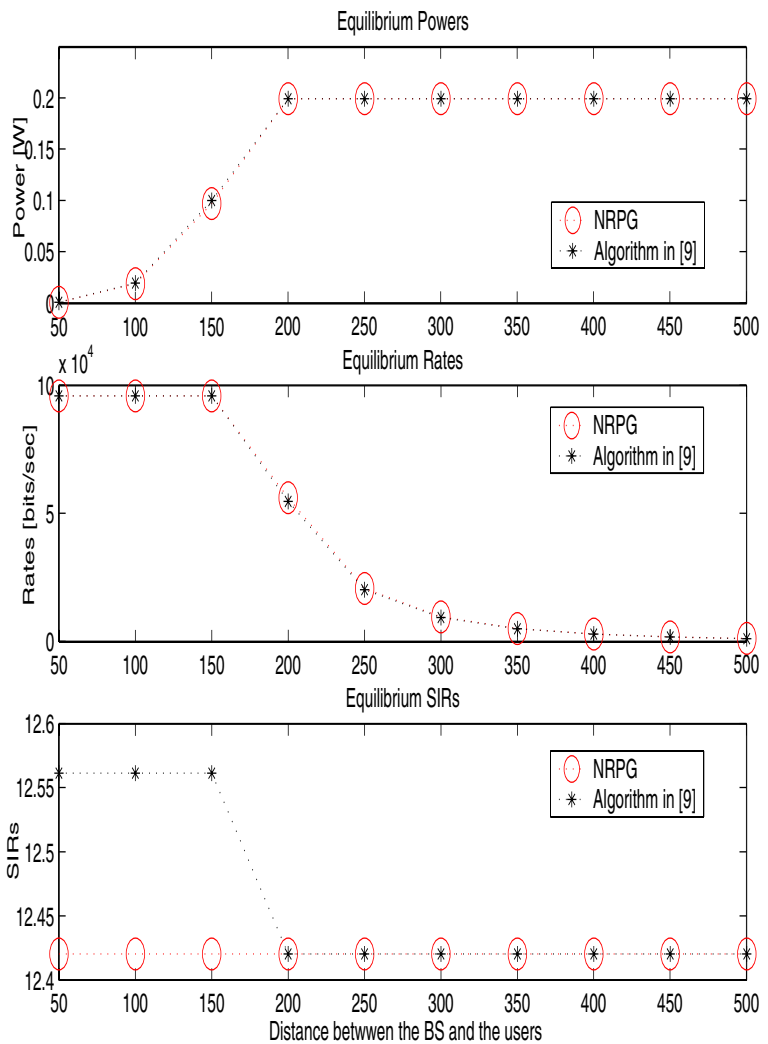

Fig. 2. Equilibrium Powers, Rates and SIRs of NRPG and algorithm in [9] closer to the base station having higher rates, lower powers, and higher SIRs than users farther away from the base station. We also note that in NRPG all the users have the same equilibrium SIR (which is desirable), whereas the equilibrium SIRs corresponding to all the users in the algorithm in [9] are different.

\section{CONCLUSION}

In this paper we applied game-theoretic concepts to model the problem of joint transmission rate and power control in a wireless system. A utility function, defined as the ratio of throughput to the transmit power was introduced. Maximization of the utility function implies optimal transmission rate and power for mobile terminals. We developed a distributed algorithm for joint rate and power control based on users maximizing their individual utilities. When transmission rates are fixed the proposed algorithm performs similar to related algorithms for power control [7], otherwise the algorithm yields the same equilibrium rates and powers as the algorithm in [9]. However, our algorithm finds the optimal rate of transmission and allocates the power required to transmit in the same step, which is different from previous approaches and implies a simpler implementation with lower complexity. Results presented in this work can be further improved by applying the concepts of pricing to the joint rate and power control problem, and we are currently working on this.

\section{ACKNOWLEDGMENT}

This work was supported in part by the National Science Foundation under grant CCR-0312323.

\section{REFERENCES}

[1] T. Alpcan, T. Basar, R. Srikant, and E. Atman. CDMA Uplink Power Control as a Noncooperative Game. In Proceedings $40^{\text {th }}$ IEEE Conference on Decision and Control, volume 1, pages 197-202, Orlando, FL, December 2001.

[2] D. J. Goodman and N. B. Mandayam. Power Control for Wireless Data. IEEE Personal Communications Magazine, 7(2):48-54, April 2000.

[3] J. C. Goodman. A Note on Existence and Uniqueness of Equilibrium Points for Concave $N$-Person Games. Econometrica, 48(1):251, 1980.

[4] S. Gunturi and F. Paganini. A Game Theoretic Approach to Power Control in Cellular CDMA. In Proceedings $58^{\text {th }}$ IEEE Vehicular Technology Conference - VTC 2003 Fall, volume 4, pages 2362-2366, Orlando, FL, October 2003.

[5] M. Hayajneh and C. T. Abdallah. Distributed joint rate and power control game-theoretic algorithms for wireless data. IEEE Communications Letters, 8(8):511-513, August 2004.

[6] J. B. Rosen. Existence and Uniqueness of Equilibrium Points for Concave $N$-Person Games. Econometrica, 33(3):520-534, 1965.

[7] C. U. Saraydar, N. B. Mandayam, and D. J. Goodman. Efficient Power Control via Pricing in Wireless Data Networks. IEEE Transactions on Communications, 50(2):291-303, February 2002.

[8] Z. Yin, J. Xie, and P. Lv. An Economic Model for Power Control in Wireless CDMA Data Networks. In Proceedings 2003 International Conference on Communication Technology - ICCT 2003, volume 2 , pages 835-838, Beijing, People's Republic of China, April 2003.

[9] W. Zhao and M. Lu. Distributed Rate and Power Control for CDMA Uplink. In Proceedings 2004 IEEE Wireless Telecommunications Symposium, pages 9-14, Pomona, CA, May 2004.

[10] L. Zhu and S. Wu. Power control for CDMA data communication systems. In Proceedings 2002 IEEE International Conference on Communications, Circuits and Systems, and West Sino Expositions, volume 1, pages 367-371, Chengdu, People's Republic of China, June 2002 . 